\title{
INEQUALITIES OF HARDY TYPE IN TWO VARIABLES
}

\section{GOU-SHENG YANG AND SHOU-PING JEAN}

\section{Introduction}

In 1920, Hardy [1] (or [3], Theorem 327) proved the following theorem:

Theorem A. If $p>1, f(x) \geq 0$ for $0<x<\infty$ and $G(x)=\frac{1}{x} \int_{0}^{x} f(t) d t$, then

$$
\int_{0}^{\infty} G^{p}(x) d x<\left(\frac{p}{p-1}\right)^{p} \int_{0}^{\infty} f^{p}(x) d x
$$

unless $f \equiv 0$. The constant is best possible.

In 1928, Hardy [2] proved the following generalization of (1.1):

Theorem $\mathbb{B}$. If $p>1, m \neq 1, f(x) \geq 0$ for $0<x<\infty$, and

$$
G(x)= \begin{cases}\frac{1}{x} \int_{0}^{x} f(t) d t, & \text { if } m>1 \\ \frac{1}{x} \int_{x}^{\infty} f(t) d t, & m<1\end{cases}
$$

then

$$
\int_{0}^{\infty} \chi^{p-m} G^{p}(x) d x<\left(\frac{p}{m-1}\right)^{p} \int_{0}^{\infty} x^{-m}(x f(x))^{p} d x, \quad \text { if } \quad m>1,
$$

and

$$
\int_{0}^{\infty} \chi^{p-m} \mathcal{G}^{p}(x) d x<\left(\frac{p}{1-m}\right)^{p} \int_{0}^{\infty} x^{-m}(x f(x))^{p} d x, \quad \text { if } \quad m<1,
$$

Received September 23, 1992; revised October 29, 1992. 
unless $f \equiv 0$, the constant is best possible.

Here functions are assumed to be measurable and left sides of inequalities exist when right sides do. In 1963, Levinson [4] proved the following generalization of (1.1):

Theoem C. For $x>0$, let $f(x) \geq 0$ and $r(x)>0$ be absolutely continuous. If $p>1$ and $\lambda>0$ so that $\frac{p-1}{p}+\frac{x r^{\prime}(x)}{r(x)} \geq \frac{1}{\lambda}$, for almost all $x>0$, then

$$
\int_{0}^{\infty} G^{p}(x) d x \leq \lambda^{p} \int_{0}^{\infty} f^{p}(x) d x
$$

where $G(x)=\frac{1}{x r(x)} \int_{0}^{x} r(t) f(t) d t$. The case $r=1$ and $\lambda=\frac{p}{p-1}$, shows the constant to be best possible. In 1986,K. L. Lee and G. S. Yang established the following generalizations:

Theorem $\mathbb{D}$. Let $p>1$ and $f, r$ be defined as in Theorem C. If there is a $\lambda>0$, such that

$$
p-1+\frac{R(x) r^{\prime}(x)}{r^{2}(x)}-\frac{(p-m) R(x)}{x r(x)} \geq \frac{p}{\lambda},
$$

for almost all $x>0$, then

$$
\int_{0}^{\infty} x^{-m+p}\left(\frac{1}{R(x)} \int_{0}^{x} r(t) f(t) d t\right)^{p} d x \leq \lambda^{p} \int_{0}^{\infty} x^{-m+p} f^{p}(x) d x, \quad m>1,
$$

where $R(x)=\int_{0}^{x} r(t) d t$.

Theorem $\mathbb{E}$. Let $p>1$ and $f, r$ be defined as in Theorem $\mathrm{C}$. If there is a $\lambda>0$ such that

$$
\frac{m-1}{p}+\frac{x r^{\prime}(x)}{r(x)} \geq \frac{1}{\lambda}
$$

for almost all $x>0$, then

$$
\int_{0}^{\infty} x^{-m+p} G^{p}(x) d x \leq \lambda^{p} \int_{0}^{\infty} x^{-m+p} f^{p}(x) d x, \quad m>1
$$

where $G(x)=\frac{1}{x r(x)} \int_{0}^{x} r(t) f(t) d t$. 
Theorem $\mathbb{F}$. Let $p>1, \alpha>1$ and $f, r$ be defined as in Theorem $\mathrm{C}$. If there is $a \lambda>0$ such that

$$
p-1-\frac{(m+\alpha p-p) R(x)}{x r(x)}-\frac{R(x) r^{\prime}(x)}{r^{2}(x)} \geq \frac{p}{\lambda}
$$

for almost all $x>0$, then

$$
\int_{0}^{\infty} x^{-m-\alpha p+p}\left(\frac{1}{R(x)} \int_{x}^{\infty} t^{\alpha} r(t) f(t) d t\right)^{p} d x \leq \lambda^{p} \int_{0}^{\infty} x^{-m+p} f^{p}(x) d x, m<1,
$$

where

$$
R(x)=\int_{x}^{\infty} r(t) d t
$$

Theorem G. Let $p>1, \alpha>1$ and $f, r$ be defined as in Theorem C. If there is $a \lambda>0$ such that

$$
\frac{1-m}{p}-\frac{x r^{\prime}(x)}{r(x)}-\alpha \geq \frac{1}{\lambda}
$$

for almost all $x>0$, then

$$
\int_{0}^{\infty} x^{-m} G^{p}(x) d x \leq \lambda^{p} \int_{0}^{\infty} x^{-m+p} f^{p}(x) d x, \quad m<1,
$$

where $G(x)=\frac{1}{x^{\alpha} r(x)} \int_{x}^{\infty} t^{\alpha} r(t) f(t) d t$.

In this paper, we shall establish inequalities similar to (1.4) - (1.7) in case the function $f$ considered is a function of two variables.

\section{The case $m>1$}

Theorem 2.1. Let $p>1, f$ be a nonnegetive integrable function defined on $\{(x, y): x>0, y>0\}$ and $q, r$ be positive absolutely continuous on $(0, \infty)$. If there are $\alpha>0, \beta>0$ such that

$$
p-1+\frac{Q(x) q^{\prime}(x)}{q^{2}(x)}-\frac{(p-m) Q(x)}{x q(x)} \geq \frac{p}{\alpha}, \quad \text { for all } x \in(0, \infty)
$$




$$
p-1+\frac{R(y) r^{\prime}(y)}{r^{2}(y)}-\frac{(p-m) R(y)}{y r(y)} \geq \frac{p}{\beta}, \quad \text { for all } y \in(0, \infty)
$$

where $Q(x)=\int_{0}^{x} q(s) d s$ and $R(y)=\int_{0}^{y} r(t) d t$, then

$$
\begin{aligned}
& \int_{0}^{\infty} \int_{0}^{\infty}(x y)^{-m+p}\left(\frac{1}{Q(x) R(y)} \int_{0}^{x} \int_{0}^{y} q(s) r(t) f(s, t) d s d t\right)^{p} d x d y \\
\leq & (\alpha \beta)^{p} \int_{0}^{\infty} \int_{0}^{\infty}(x y)^{-m+p} f^{p}(x, y) d x d y .
\end{aligned}
$$

Proof. If $0<a<b, 0<c<d$, let

$$
I(x, y)=\int_{a}^{x} \int_{c}^{y} q(s) r(t) f(s, t) d s d t x \in[a, b], y \in[c, d] .
$$

Then

$$
\begin{aligned}
& \int_{a}^{b} \int_{c}^{d}(x y)^{-m+p}\left(\frac{1}{Q(x) R(y)} \int_{a}^{x} \int_{c}^{y} q(s) r(t) f(s, t) d s d t\right)^{p} d x d y \\
= & \int_{a}^{b}\left[\int_{c}^{d}(x y)^{-m+p}(Q(x) R(y))^{-p} I^{p}(x, y) d x\right] d y \\
= & \int_{c}^{d} y^{-m+p} R^{-p}(y)\left[\int_{a}^{b} x^{-m+p} Q^{-p}(x) I^{p}(x, y) d x\right] d y .
\end{aligned}
$$

Fix $y$ and integration by parts, we have

$$
\begin{aligned}
& \int_{a}^{b} x^{-m+p} Q^{-p}(x) I^{p}(x, y) d x=\left.\frac{Q^{-p+1}(x) x^{-m+p}}{(-p+1) q(x)} I^{p}(x, y)\right|_{a} ^{b} \\
& +\frac{1}{p-1} \int_{a}^{b} x^{-m+p} Q^{-p}(x) I^{p}(x, y)\left[-\frac{Q(x) q^{\prime}(x)}{q^{2}(x)}+\frac{(p-m) Q(x)}{x q(x)}\right] d x \\
& +\frac{p}{p-1} \int_{a}^{b} x^{-m+p} Q^{-p+1}(x) I^{p-1}(x, y)\left(\int_{c}^{y} r(t) f(x, t) d t\right) d x .
\end{aligned}
$$

so that

$$
\begin{aligned}
& \int_{a}^{b} x^{-m+p} Q^{-p}(x) I^{p}(x, y)\left[p-1+\frac{Q(x) q^{\prime}(x)}{q^{2}(x)}-\frac{(p-m) Q(x)}{x q(x)}\right] d x \\
\leq & p \int_{a}^{b} x^{-m+p} Q^{-p+1}(x) I^{p-1}(x, y)\left(\int_{c}^{y} r(t) f(x, t) d t\right) d x .
\end{aligned}
$$


Using (2.1) and Holder inequality with indices $p$ and $p /(p-1)$, we have

$$
\begin{aligned}
& \int_{a}^{b} x^{-m+p} Q^{-p}(x) I^{p}(x, y) d x \\
\leq & \alpha \int_{a}^{b}\left(x^{-m+p} Q^{-p}(x) I^{p}(x, y)\right)^{\frac{p-1}{p}}\left[x^{-m+p}\left(\int_{c}^{y} r(t) f(x, t) d t\right)^{p} d x\right]^{\frac{1}{p}} \\
\leq & \alpha\left[\int_{a}^{b} x^{-m+p} Q^{-p}(x) I^{p}(x, y) d x\right]^{\frac{p-1}{p}}\left[\int_{a}^{b} x^{-m+p}\left(\int_{c}^{y} r(t) f(x, t) d t\right)^{p} d x\right]^{\frac{1}{p}} .
\end{aligned}
$$

It follows that $\int_{a}^{b} x^{-m+p} Q^{-p}(x) I^{p}(x, y) d x \leq \alpha^{p} \int_{a}^{b} x^{-m+p}\left(\int_{c}^{y} r(t) f(x, t) d t\right)^{p} d x$. Substituting this inequality in (2.4) and using Fubini's theorem, we have

$$
\begin{aligned}
& \int_{c}^{d} y^{-m+p} R^{-p}(y)\left[\int_{a}^{b} x^{-m+p} Q^{-p}(x) I^{p}(x, y) d x\right] d y \\
\leq & \alpha^{p} \int_{c}^{d} y^{-m+p} R^{-p}(y)\left[\int_{a}^{b} x^{-m+p}\left(\int_{c}^{y} r(t) f(x, t) d t\right)^{p} d x\right] d y \\
= & \alpha^{p} \int_{a}^{b} x^{-m+p}\left[\int_{c}^{d} y^{-m+p} R^{-p}(y)\left(\int_{c}^{y} r(t) f(x, t) d t\right)^{p} d y\right] d x .
\end{aligned}
$$

Fix $x$ and apply Theorem $D$, we have

$$
\int_{c}^{d} y^{-m+p} \mathbb{R}^{-p}\left(\int_{c}^{y} r(t) f(x, t) d t\right)^{p} d y \leq \beta^{p} \int_{c}^{d} y^{-m+p} f^{p}(x, y) d y .
$$

Using this inequality in (2.6), we obtain

$$
\begin{aligned}
& \int_{a}^{b} \int_{c}^{d}(x y)^{-m+p}(Q(x) R(y))^{-p} \mathbb{I}^{p}(x, y) d x d y \\
\leq & (\alpha, \beta)^{p} \int_{a}^{b} \int_{c}^{d}(x y)^{-m+p} f^{p}(x, y) d x d y \\
\leq & (\alpha, \beta)^{p} \int_{0}^{\infty} \int_{0}^{\infty}(x y)^{-m+p} f^{p}(x, y) d x d y .
\end{aligned}
$$

Let $a<s<b$ and $c<t<d$. Then

$$
\begin{aligned}
& \int_{s}^{b} \int_{t}^{d}(x y)^{-m+p}(Q(x) R(y))^{-p} I^{p}(x, y) d x d y \\
\leq & (\alpha \beta)^{p} \int_{0}^{\infty} \int_{0}^{\infty}(x y)^{-m+p} f^{p}(x, y) d x d y .
\end{aligned}
$$


Let $a \rightarrow 0$ and $c \rightarrow 0$, we see that

$$
\begin{aligned}
& \int_{s}^{b} \int_{t}^{d}(x y)^{-m+p}\left(\frac{I}{Q(x) R(y)} \int_{0}^{x} \int_{0}^{y} q(s) r(t) f(s, t) d s d t\right)^{p} d x d y \\
\leq & (\alpha \beta)^{p} \int_{0}^{\infty} \int_{0}^{\infty}(x y)^{-m+p} f^{p}(x, y) d x d y .
\end{aligned}
$$

holds for all $s, t, b, d$ such that $0<s<b$ and $0<t<d$, the required inequality then follows.

Theorem 2.2. Let $p>1$ and $f, g, r$ be defined as in Theorem 2.1. If there are $\alpha>0, \beta>0$ such that

$$
\begin{aligned}
& \frac{m-1}{p}+\frac{x q^{\prime}(x)}{q(x)} \geq \frac{1}{\alpha}, \quad \text { for all } x \in(0, \infty), \\
& \frac{m-1}{p}+\frac{y r^{\prime}(y)}{r(y)} \geq \frac{1}{\beta}, \text { for all } y \in(0, \infty),
\end{aligned}
$$

then

$$
\begin{aligned}
& \int_{0}^{\infty} \int_{0}^{\infty}(x y)^{-m+p}\left(\frac{1}{x y q(x) r(y)} \int_{0}^{x} \int_{0}^{y} q(s) r(t) f(s, t) d s d t\right)^{p} d x d y \\
\leq & (\alpha \beta)^{p} \int_{0}^{\infty} \int_{0}^{\infty}(x y)^{-m+p} f^{p}(x, y) d x d y .
\end{aligned}
$$

Proof. If $0>a>b, 0<c<d$, let

$$
I(x, y)=\int_{0}^{x} \int_{0}^{y} q(s) r(t) f(s, t) d s d t, x \in[a, b], y \in[c, d]
$$

then

$$
\begin{aligned}
& \int_{a}^{b} \int_{c}^{d}(x y)^{-m+p}\left(\frac{1}{x y q(x) r(y)} \int_{a}^{x} \int_{c}^{y} q(s) r(t) f(s, t) d s d t\right)^{p} d x d y \\
= & \int_{a}^{b}\left[\int_{c}^{d}(x y)^{-m+p}\left(\frac{1}{x y q(x) r(y)}\right)^{p} I^{p}(x, y) d x\right] d y \\
= & \int_{c}^{d} y^{-m} r^{-p}(y)\left[\int_{c}^{b} x^{-m} q^{-p}(x) I^{p}(x, y) d x\right] d y .
\end{aligned}
$$


Fix $y$, integration by parts gives

$$
\begin{aligned}
& \int_{a}^{b} x^{-m} q^{-p}(x) I^{p}(x, y) d x=\left.\frac{x^{-m+1}}{-m+1} q^{-p}(x) I^{p}(x, y)\right|_{a} ^{b} \\
& +\frac{p}{m-1} \int_{a}^{b} x^{-m} q^{-p}(x) I^{p}(x, y)\left(\frac{-x q^{\prime}(x)}{q(x)}\right) d x \\
& +\frac{p}{m-1} \int_{a}^{b} x^{-m+1} q^{-p+1}(x) I^{p-1}(x, y)\left(\int_{c}^{y} r(t) f(x, t) d t\right) d x .
\end{aligned}
$$

so that

$$
\begin{aligned}
& \int_{a}^{b} x^{-m} q^{-p}(x) I^{p}(x, y)\left[\frac{m-1}{p}+\frac{x q^{\prime}(x)}{q(x)}\right] d x \\
\leq & \int_{a}^{b} x^{-m+1} q^{-p+1}(x) I^{p-1}(x, y)\left(\int_{c}^{y} r(t) f(x, t) d t\right) d x .
\end{aligned}
$$

Applying (2.7) and Holder inequality with indices $p$ and $p /(p-1)$, we have

$$
\begin{aligned}
& \int_{a}^{b} x^{-m} q^{-p}(x) I^{p}(x, y) d x \\
\leq & \alpha \int_{a}^{b}\left(x^{-m} q^{-p}(x) I^{p}(x, y)\right)^{\frac{p-1}{p}}\left[x^{-m+p}\left(\int_{c}^{y} r(t) f(x, t) d t\right)^{p}\right]^{\frac{1}{p}} d x \\
\leq & \alpha\left[\int_{a}^{b} x^{-m} q^{-p}(x) I^{p}(x, y) d x\right]^{\frac{p-1}{p}}\left[\int_{a}^{b} x^{-m+p}\left(\int_{c}^{y} r(t) f(x, t) d t\right)^{p} d x\right]^{\frac{1}{p}} .
\end{aligned}
$$

It follows that

$$
\int_{a}^{b} x^{-m} q^{-p}(x) I^{p}(x, y) d x \leq \alpha^{p} \int_{a}^{b} x^{-m+p}\left(\int_{c}^{y} r(t) f(x, t) d t\right)^{p} d x .
$$

Substituting this inequality in (2.10) and using Fubini's theorem, we have

$$
\begin{aligned}
& \int_{c}^{d} y^{-m} r^{-p}(y)\left[\int_{a}^{b} x^{-m} q^{-p}(x) I^{p}(x, y) d x\right] d y \\
\leq & \alpha^{p} \int_{c}^{d} y^{-m} r^{-p}(y)\left[\int_{a}^{b} x^{-m+p}\left(\int_{c}^{y} r(t) f(x, t) d t\right)^{p} d x\right] d y \\
= & \alpha^{p} \int_{a}^{b} x^{-m+p}\left[\int_{c}^{d} y^{-m} r^{-p}(y)\left(\int_{c}^{y} r(t) f(x, t) d t\right)^{p} d y\right] d x .
\end{aligned}
$$


Now fix $x$ and use Theorem $E$, we have

$$
\int_{c}^{d} y^{-m} r^{-p}(y)\left(\int_{c}^{y} r(t) f(x, t) d t\right)^{p} d y \leq \beta^{p} \int_{c}^{d} y^{-m+p} f^{p}(x, y) d y .
$$

Substituting this inequality in (2.12), we have

$$
\begin{aligned}
& \int_{a}^{b} \int_{c}^{d}(x y)^{-m}\left(\frac{1}{q(x) r(y)}\right)^{p} I^{p}(x, y) d x d y \\
\leq & (\alpha \beta)^{p} \int_{a}^{b} \int_{c}^{d}(x y)^{-m+p} f^{p}(x, y) d x d y \\
\leq & (\alpha \beta)^{p} \int_{0}^{\infty} \int_{0}^{\infty}(x y)^{-m+p} f^{p}(x, y) d x d y .
\end{aligned}
$$

Let $a<s<b$ and $c<t<d$. Then

$$
\begin{aligned}
& \int_{s}^{b} \int_{t}^{d}(x y)^{-m}\left(\frac{1}{q(x) r(y)}\right)^{p} I^{p}(x, y) d x d y \\
\leq & (\alpha \beta)^{p} \int_{0}^{\infty} \int_{0}^{\infty}(x y)^{-m+p} f^{p}(x, y) d x d y .
\end{aligned}
$$

Let $a \rightarrow 0$ and $c \rightarrow 0$. We see that

$$
\begin{aligned}
& \int_{s}^{b} \int_{t}^{d}(x y)^{-m+p}\left(\frac{1}{x y q(x) r(y)} \int_{0}^{x} \int_{0}^{y} q(s) r(t) f(s, t) d s d t\right)^{p} d x d y \\
\leq & (\alpha \beta)^{p} \int_{0}^{\infty} \int_{0}^{\infty}(x y)^{-m+p} f^{p}(x, y) d x d y .
\end{aligned}
$$

holds for all $s, b, t, d$ such that $0<s<b, 0<t<d$. The required inequality then follows.

\section{The case $m<1$}

Theorem 3.1. Let $p>1, \lambda>1$ and $f$ be a nonnegative integrable function on $\{(x, y): x>0, y>0\}$ and $q, r$ be positive absolutely continuous functions on $(0, \infty)$. If there are $\alpha>0, \beta>0$ such that

$$
p-1-\frac{(m+\lambda p-p) Q(x)}{x q(x)}-\frac{Q(x) q^{\prime}(x)}{q^{2}(x)} \geq \frac{p}{\alpha}, \text { for all } x \in(0, \infty),
$$




$$
p-1-\frac{(m+\lambda p-p) R(y)}{y r(y)}-\frac{R(y) r^{\prime}(y)}{r^{2}(y)} \geq \frac{p}{\beta}, \quad \text { for all } y \in(0, \infty),
$$

where $Q(x)=\int_{x}^{\infty} q(s) d s$ and $R(y)=\int_{y}^{\infty} r(t) d t$, then

$$
\begin{aligned}
& \int_{0}^{\infty} \int_{0}^{\infty}(x y)^{-m-\lambda p+p}\left(\frac{1}{Q(x) R(y)} \int_{x}^{\infty} \int_{y}^{\infty}(s t)^{\lambda} q(s) r(t) f(s, t) d s d t\right)^{p} d x d y \\
\leq & (\alpha \beta)^{p} \int_{0}^{\infty} \int_{0}^{\infty}(x y)^{-m+p} f^{p}(x, y) d x d y .
\end{aligned}
$$

Proof. If $0<a<b, 0<c<d$, let

$$
I(x, y)=\int_{x}^{b} \int_{y}^{d}(s t)^{\lambda} q(s) r(t) f(s, t) d s d t \quad x \in[a, b], \quad y \in[c, d]
$$

Then

$$
\begin{aligned}
& \int_{a}^{b} \int_{c}^{d}(x y)^{-m-\lambda p+p}\left(\frac{1}{Q(x) R(y)} \int_{x}^{b} \int_{y}^{d}(s t)^{\lambda} q(s) r(t) f(s, t) d s d t\right)^{p} d x d y \\
= & \int_{a}^{b}\left[\int_{c}^{d}(x y)^{-m-\lambda p+p}(Q(x) R(y))^{-p} I^{p}(x, y) d x\right] d y \\
= & \int_{c}^{d} y^{-m-\lambda p+p} R^{-p}(y)\left[\int_{a}^{b} x^{-m-\lambda p+p} Q^{-p}(x) I^{p}(x, y) d x\right] d y .
\end{aligned}
$$

Fix $y$ and integration by parts, we have

$$
\begin{aligned}
& \int_{a}^{b} x^{-m-\lambda p+p} Q^{-p}(x) I^{p}(x, y) d x=\left.\frac{Q^{-p+1}(x) x^{-m-\lambda p+p}}{(p-1) q(x)} I^{p}(x, y)\right|_{a} ^{b} \\
& -\frac{1}{p-1} \int_{a}^{b} x^{-m-\lambda p+p} Q^{-p}(x) I^{p}(x, y)\left[\frac{(-m-\lambda p+p) Q(x)}{q(x) x}-\frac{Q(x) q^{\prime}(x)}{q^{2}(x)}\right] d x \\
& +\frac{p}{p-1} \int_{a}^{b} x^{-m-\lambda p+p} x^{\lambda} Q^{-p+1}(x)\left(\int_{y}^{d} t^{\lambda} r(t) f(x, t) d t\right) I^{p-1}(x, y) d x .
\end{aligned}
$$

so that

$$
\begin{aligned}
& \int_{a}^{b} x^{-m-\lambda p+p} Q^{-p}(x) I^{p}(x, y)\left[p-1-\frac{(m+\lambda p-p) Q(x)}{x q(x)}-\frac{Q(x) q^{\prime}(x)}{q^{2}(x)}\right] d x \\
\leq & p \int_{a}^{b} x^{-m-\lambda p+p} x^{\lambda} Q^{-p+1}(x)\left(\int_{y}^{d} t^{\lambda} r(t) f(x, t) d t\right) I^{p-1}(x, y) d x .
\end{aligned}
$$


Using (3.1) and Holder inequality to obtain

$$
\begin{aligned}
& \int_{a}^{b} x^{-m-\lambda p+p} Q^{-p}(x) I^{p}(x, y) d x \\
\leq & \alpha \int_{a}^{b}\left(x^{-m-\lambda p+p} Q^{-p}(x) I^{p}(x, y)\right)^{\frac{p-1}{p}}\left[x^{-m-\lambda p+p} x^{\lambda p}\left(\int_{y}^{d} t^{\lambda} r(t) f(x, t) d t\right)^{p}\right]^{\frac{1}{p}} d x \\
\leq & \alpha\left[\int_{a}^{b} x^{-m-\lambda p+p} Q^{-p}(x) I^{p}(x, y) d x\right]^{\frac{p-1}{p}}\left[\int_{a}^{b} x^{-m+p}\left(\int_{y}^{d} t^{\lambda} r(t) f(x, t) d t\right)^{p} d x\right]^{\frac{1}{p}} .
\end{aligned}
$$

so that

$$
\int_{a}^{b} x^{-n-\lambda p+p} Q^{-p}(x) I^{p}(x, y) d x \leq \alpha^{p} \int_{a}^{b} x^{-m+p}\left(\int_{y}^{d} t^{\lambda} r(t) f(x, t) d t\right)^{p} d x .
$$

Substituting this inequality in (3.4) and using Fubini's theorem, we have

$$
\begin{aligned}
& \int_{c}^{d} y^{-m-\lambda p+p} R^{-p}(y)\left[\int_{a}^{b} x^{-m-\lambda p+p} Q^{-p}(x) I^{p}(x, y) d x\right] d y \\
\leq & \alpha^{p} \int_{c}^{d} y^{-m-\lambda p+p} R^{-p}(y)\left[\int_{a}^{b} x^{-m+p}\left(\int_{y}^{d} t^{\lambda} r(t) f(x, t) d t\right)^{p} d x\right] d y \\
= & \alpha^{p} \int_{a}^{b} x^{-m+p}\left[\int_{c}^{d} y^{-m-\lambda p+p} R^{-p}(y)\left(\int_{y}^{d} t^{\lambda} r(t) f(x, t) d t\right)^{p} d y\right] d x .
\end{aligned}
$$

Fix $x$ and use Theorem $\mathrm{F}$, we have

$$
\int_{c}^{d^{l}} y^{-m-\lambda p+p} R^{-p}(y)\left(\int_{y}^{d} t^{\lambda} r(t) f(x, t) d t\right)^{p} d y \leq \beta^{p} \int_{c}^{d} y^{-m+p} f^{p}(x, y) d y .
$$

Substituting this inequality in (3.6), we have

$$
\begin{aligned}
& \int_{a}^{b} \int_{c}^{d}(x y)^{-m-\lambda p+p}(Q(x) R(y))^{-p} I^{p}(x, y) d x d y \\
\leq & (\alpha \beta)^{p} \int_{a}^{b} \int_{c}^{d}(x y)^{-m+p} f^{p}(x, y) d x d y \\
\leq & (\alpha \beta)^{p} \int_{0}^{\infty} \int_{0}^{\infty}(x y)^{-m+p} f^{p}(x, y) d x d y .
\end{aligned}
$$


Let $a<s<b$ and $c<t<d$. Then

$$
\begin{aligned}
& \int_{a}^{s} \int_{c}^{t}(x y)^{-m-\lambda p+p}(Q(x) R(y))^{-p} I^{p}(x, y) d x d y \\
\leq & (\alpha \beta)^{p} \int_{0}^{\infty} \int_{0}^{\infty}(x y)^{-m+p} f^{p}(x, y) d x d y .
\end{aligned}
$$

Let $b \rightarrow \infty$ and $d \rightarrow \infty$ we see that

$$
\begin{aligned}
& \int_{a}^{s} \int_{c}^{t}(x y)^{-m-\lambda p+p}\left(\frac{1}{Q(x) R(y)} \int_{x}^{\infty} \int_{y}^{\infty}(s t)^{\lambda} q(s) r(t) f(s, t) d s d t\right)^{p} d x d y \\
\leq & (\alpha \beta)^{p} \int_{0}^{\infty} \int_{0}^{\infty}(x y)^{-m+p} f^{p}(x, y) d x d y,
\end{aligned}
$$

holds for all $a, s, c, t$ such that $0<a<s, 0<c<t$, the required inequality then follows.

Theorem 3.2. Let $p, \lambda$ and $f, r$ be defined as in Theorem 3.1, If there are $\alpha>0, \beta>0$, such that

$$
\begin{aligned}
& \frac{1-m}{p}-\frac{x q^{\prime}(x)}{q(x)}-\lambda \geq \frac{1}{\alpha}, \text { for all } x \in(0, \infty) \\
& \frac{1-m}{p}-\frac{y r^{\prime}(y)}{r(y)}-\lambda \geq \frac{1}{\beta}, \text { for all } y \in(0, \infty)
\end{aligned}
$$

then

$$
\begin{aligned}
& \int_{0}^{\infty} \int_{0}^{\infty}(x y)^{-m} H^{p}(x, y) d x d y \\
\leq & (\alpha \beta)^{p} \int_{0}^{\infty} \int_{0}^{\infty}(x y)^{-m+p} f^{p}(x, y) d x d y .
\end{aligned}
$$

where

$$
H(x, y)=\frac{1}{(x y)^{\lambda} q(x) r(y)} \int_{x}^{\infty} \int_{y}^{\infty}(s t)^{\lambda} q(s) r(t) f(s, t) d s d t
$$

Proof. If $0<a<b, 0<c<d$, let

$$
I(x, y)=\int_{x}^{b} \int_{y}^{d}(s t)^{\lambda} q(s) r(t) f(s, t) d s d t, \quad x \in[a, b], y \in[c, d],
$$


Then

$$
\begin{aligned}
& \int_{a}^{b} \int_{c}^{d}(x y)^{-m}\left(\frac{1}{(x y)^{\lambda} q(x) r(y)} \int_{x}^{b} \int_{y}^{d}(s t)^{\lambda} q(s) r(t) f(s, t) d s d t\right)^{p} d x d y \\
= & \int_{a}^{b} \int_{c}^{d}(x y)^{-m}\left(\frac{1}{(x y)^{\lambda} g(x) r(y)}\right)^{p} I^{p}(x, y) d x d y \\
= & \int_{c}^{d} y^{-m}\left(\frac{1}{y^{\lambda} r(y)}\right)^{p}\left[\int_{a}^{b} x^{-m}\left(\frac{1}{x^{\lambda} q(x)}\right)^{p} I^{p}(x, y) d x\right] d y .
\end{aligned}
$$

Fix $y$ and integration by parts gives

$$
\begin{aligned}
& \int_{a}^{b} x^{-m}\left(\frac{1}{x^{\lambda} q(x)}\right)^{p} I^{p}(x, y) d x=\left.\frac{x^{-m+1}}{-m+1}\left(\frac{1}{x^{\lambda} q(x)}\right)^{p} I^{p}(x, y)\right|_{a} ^{b} \\
& +\frac{p}{m-1} \int_{a}^{b} x^{-m}\left(\frac{1}{x^{\lambda} q(x)}\right)^{p} I^{p}(x, y)\left(-\lambda+\frac{-x q^{\prime}(x)}{q(x)}\right) d x \\
& +\frac{-p}{m-1} \int_{a}^{b} x^{-m}\left(\frac{1}{x^{\lambda} q(x)}\right)^{p-1} I^{p-1}(x, y)\left(x \int_{y}^{d} t^{\lambda} r(t) f(x, t) d t\right) d x .
\end{aligned}
$$

so that

$$
\begin{aligned}
& \int_{a}^{b} x^{-m}\left(\frac{1}{x^{\lambda} q(x)}\right)^{p} I^{p}(x, y)\left[\frac{1-m}{p}-\frac{x q^{\prime}(x)}{q(x)}-\lambda\right] d x \\
\leq & \int_{a}^{b} x^{-m}\left(\frac{1}{x^{\lambda} q(x)}\right)^{p-1} I^{p-1}(x, y)\left(x \int_{y}^{d} t^{\lambda} r(t) f(x, t) d t\right) d x .
\end{aligned}
$$

Applying (3.7) and Holder inequality to obtain

$$
\int_{a}^{b} x^{-m}\left(\frac{1}{x^{\lambda} q(x)}\right)^{p} I^{p}(x, y) d x
$$

$\leq \alpha \int_{a}^{b}\left[x^{-m}\left(\frac{1}{x^{\lambda} q(x)}\right)^{p} I^{p}(x, y)\right]^{\frac{p-1}{p}}\left[x^{-m+p}\left(\int_{y}^{d} t^{\lambda} r(t) f(x, t) d t\right)^{p}\right]^{\frac{1}{p}} d x$ $\leq \alpha\left[\int_{a}^{b} x^{-m}\left(\frac{1}{x^{\lambda} q(x)}\right)^{p} I^{p}(x, y) d x\right]^{\frac{p-1}{p}}\left[\int_{a}^{b} x^{-m+p}\left(\int_{y}^{d} t^{\lambda} r(t) f(x, t) d t\right)^{p} d x\right]^{\frac{1}{p}}$.

so that

$$
\int_{a}^{b} x^{-m}\left(\frac{1}{x^{\lambda} q(x)}\right)^{p} I^{p}(x, y) d x \leq \alpha^{p} \int_{a}^{b} x^{-m+p}\left(\int_{y}^{d} t^{\lambda} r(t) f(x, t) d t\right)^{p} d x .
$$


Substituting this inequality in (3.10) and using Fubini's theorem, we have

$$
\begin{aligned}
& \int_{c}^{d} y^{-m}\left(\frac{1}{y^{\lambda} r(y)}\right)^{p}\left[\int_{a}^{b} x^{-m}\left(\frac{1}{x^{\lambda} q(x)}\right) I^{p}(x, y) d x\right] d y \\
\leq & \alpha^{p} \int_{c}^{d} y^{-m}\left(\frac{1}{y^{\lambda} r(y)}\right)^{p}\left[\int_{a}^{b} x^{-m+p}\left(\int_{y}^{d} t^{\lambda} r(t) f(x, t) d t\right)^{p} d x\right] d y \\
= & \alpha^{p} \int_{a}^{b} x^{-m+p}\left[\int_{c}^{d} y^{-m}\left(\frac{1}{y^{\lambda} r(y)}\right)^{p}\left(\int_{y}^{d} t^{\lambda} r(t) f(x, t) d t\right)^{p} d y\right] d x .
\end{aligned}
$$

Fix $x$ and use Theorem $\mathrm{G}$, we have

$$
\int_{c}^{d} y^{-m}\left(\frac{1}{y^{\lambda} r(y)}\right)^{p}\left(\int_{y}^{d} t^{\lambda} r(t) f(x, t) d t\right)^{p} d y \leq \beta^{p} \int_{c}^{d} y^{-m+p} f^{p}(x, y) d y .
$$

Substituting this inequality in (3.15), we have

$$
\begin{aligned}
& \int_{a}^{b} \int_{c}^{d}(x y)^{-m}\left(\frac{1}{(x y)^{\lambda} q(x) r(y)}\right)^{p} I^{p}(x, y) d x d y \\
\leq & (\alpha \beta)^{p} \int_{a}^{b} \int_{c}^{d}(x y)^{-m+p} f^{p}(x, y) d x d y \\
\leq & (\alpha \beta)^{p} \int_{0}^{\infty} \int_{0}^{\infty}(x y)^{-m+p} f^{p}(x, y) d x d y .
\end{aligned}
$$

Let $a<s<b$ and $c<t<d$. Then

$$
\begin{aligned}
& \int_{a}^{s} \int_{c}^{t}(x y)^{-m}\left(\frac{1}{(x y)^{\lambda} q(x) r(y)}\right)^{p} I^{p}(x, y) d x d y \\
\leq & (\alpha \beta)^{p} \int_{0}^{\infty} \int_{0}^{\infty}(x y)^{-m+p} f^{p}(x, y) d x d y .
\end{aligned}
$$

Let $b \rightarrow \infty$ and $d \rightarrow \infty$ we see that

$$
\begin{aligned}
& \int_{a}^{s} \int_{c}^{d}(x y)^{-m} H^{p}(x, y) d x d y \\
\leq & (\alpha \beta)^{p} \int_{0}^{\infty} \int_{0}^{\infty}(x y)^{-m+p} f^{p}(x, y) d x d y,
\end{aligned}
$$

holds for all $a, s, c, t$ such that $0<a<s, 0<c<t$, the required inequality then follows. 


\section{References}

[1] G. H. Hardy, "Note on a theorem of Hibert", Math. Zeit, 6 (1920), 314-317.

[2] G. H. Hardy, "Note on some points in the integral calculus", Messenger of Math., 57 (1982), 12-16.

[3] G. H. Hardy, J. E. Littlewood and Pōlya, "Inequalities", 2nd Edition, Cambridge, 1952.

[4] N. Levinson, "Generalization of an inequality of Hardy", Duck Math. J. 31 (1964), 389394.

[5] Kin-Chun Lee and Cou-Sheng Yang, "On generalization of Hardy's inequality", Tamkang Journal of Mathematics, Vol. 17, No. 4, 1986, pp. 109-119.

Department of mathematics, Tamkang University, Tamsui, Taiwan. 